\title{
Observation of Low Frequency Oscillations in GaAs Samples Grown by Molecular Beam Epitaxy at Low Temperatures
}

\author{
R. M. Rubinger, A. G. de Oliveira, G. M. Ribeiro, D. A. W. Soares*, \\ and M. V. B. Moreira \\ Departamento de Física, Universidade Federal de Minas Gerais \\ CP 702, 30123-970 Belo Horizonte, Brazil
}

Received February, 1999

\begin{abstract}
We have observed Low Frequency Oscillations (LFO) in a GaAs sample grown by molecular beam epitaxy (MBE) at $300^{\circ} \mathrm{C}$ as a function of applied electric field, temperature and illumination intensity. Time series of the oscillations for different values of the applied electric field at room temperature were obtained. We have also obtained the $\mathbf{j x E}$ characteristics as a function of temperature and light intensity. We were able to control the LFO using three experimental parameters, the electrical field, which controls the oscillation frequency, and the temperature and illumination intensity, which control the carrier density and field-enhanced trapping. We were able to identify the LFO dependence on the carrier density and on the field-enhanced trapping.
\end{abstract}

\section{Introduction}

Low frequency oscillations (LFO) are detected in the external current of semiconductor devices in the range of sub- $\mathrm{Hz}$ to few hundreds $\mathrm{Hz}$. Inside the device they constitute self-organized spatio-temporal structures of the type of electric field domains moving from the cathode to the anode. Because of their low frequency oscillations are easy to be observed and analyzed since no sophisticated instrumentation is needed in order to carry out measurements. It is also worth to mention that semiconductors are one of the most easily reproducible systems for the study of nonlinear dynamics and deterministic chaotic phenomena. In this wayCLFO in semi-insulating (SI) material are a good theme for such studies.

We have studied the $\mathbf{j x} \mathbf{E}$ characteristics of a GaAs sample grown by MBE at $300^{\circ} \mathrm{C}$ looking for LFO and routes to chaos of the LFO [12 [3]. In semiconductors presenting negative differential conductivity (NDC) $\Gamma$ moving high-field domains can build up spontaneously leading to current oscillations. Such oscillations occur due to the effect of field enhanced trapping of carriers been captured into deep traps. The low temperature MBE growth (LT-MBE) of GaAs leads to a high density of intrinsic defectsT mainly in the form of antisite defects nnamely As atoms in the Ga site $\left(\mathrm{As}_{G a}\right)$. These defects are efficient in inducing field enhanced trapping. For this work typical samples were cut in small rectangles and two indium contacts were defined separated in about $200 \mu \mathrm{m}$. Samples grown at low temperatures

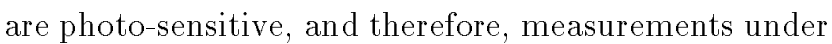
illumination using an infrared LED were carried out. The frequency of the oscillations is a strong function of temperature and illumination intensity. We have observed the presence of LFO in the range of sub- Hz to few $\mathrm{Hz}$ for external electrical fields ranging from $\mathrm{Vcm}^{-1}$ to $\mathrm{kVcm}^{-1}$. The definition of the intrinsic defect directly involved with LFO has been historically a hard job but our experimental setup and the sample provided favorably simple conditions to study it $\Gamma$ since we have three control parameters $\Gamma$ namely $\Gamma$ the applied voltage $\Gamma$ the temperature and the light intensity. These experimental parameters are easy to control with high accuracy and $\Gamma$ in this way we expect to be able to tune route to chaos.

The analysis of the temperature dependence of LFO has been used by other authors in order to identify deep levels in SI crystals [4]. Particularly the Arrhenius plots of the frequency modes of the LFO have been used to obtain information for GaAs [5] and InP [4] and the authors claim that this procedure has many advantages over others. A model that describes completely the phenomenology is not yet available but some ap-

\footnotetext{
*EFEI Caixa Postal 50 CEP: 37.500-000, Itajubá, Brazil
} 
proaches have been proposed based on the effects of field-enhanced trapping [5] Tfield-enhanced emission [3] and negative differential mobility [6].

In this paper we study the LFO in a LT-MBE GaAs sample. These samples presents high density of deep level defects $\Gamma$ which gives them semi-insulating (SI) characteristic since the Fermi level is pinned around the middle of the gap. Under illumination intrinsic defects release carriers into the conduction and valence bands. At room temperature $\Gamma$ we have measured the dependence of the LFO and its characteristic frequencies as a function of the electric field. The temperature and the illumination dependence of our $\mathbf{j} \mathbf{x E}$ characteristics give evidences of field enhanced trapping of free carriers in both the valence and conduction bands is the most probably model.

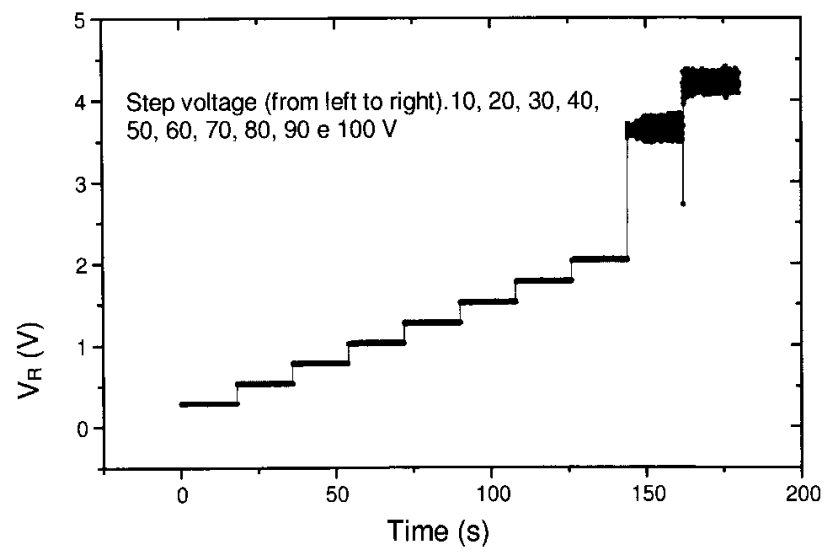

Figure 1. LFO as a function of time and applied bias. Each step corresponds to a bias, which is $10 \mathrm{~V}$ in the left and increases $10 \mathrm{~V}$ for each step to the right. The $\mathrm{Y}$ axis is the voltage through a $1 \mathrm{M} \Omega$ series resistor.

\section{Results}

The electric field dependence of the LFO observed at room temperature is shown in Fig.1. We can see that the amplitude of the oscillations increases with the value of the applied electric field. For measurements carried out with an electric field of $1500 \mathrm{Vcm}^{-1}$ (90V) and $1670 \mathrm{Vcm}^{-1}(100 \mathrm{~V})$ the increase in the amplitude of the LFO signal is more remarkable. This is explained based on the observed fact that the sample has an enormous enhancement in the free carrier density due to impurity breakdown in shallow acceptors. In Figs. 2 and 3 we show the LFO obtained at $330 \mathrm{Vcm}^{-1}(20 \mathrm{~V})$ and $1500 \mathrm{Vcm}^{-1}(90 \mathrm{~V})$. The signal obtained at 330 $\mathrm{Vcm}^{-1}$ is clearly a combination of two frequencies with the main frequency centered in $2.35 \mathrm{~Hz}$. At $1500 \mathrm{Vcm}^{-1}$ we can observe an increase in the amplitude and frequency which is about $4.3 \mathrm{~Hz}$.

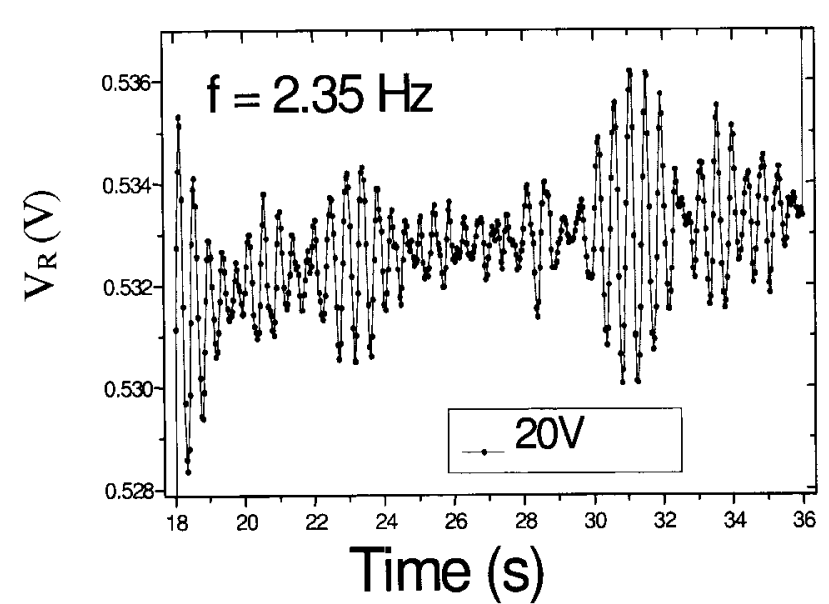

Figure 2. A zoom of Fig. 1 for the step of $20 \mathrm{~V}$. Observe that the main frequency is of $2.35 \mathrm{~Hz}$.

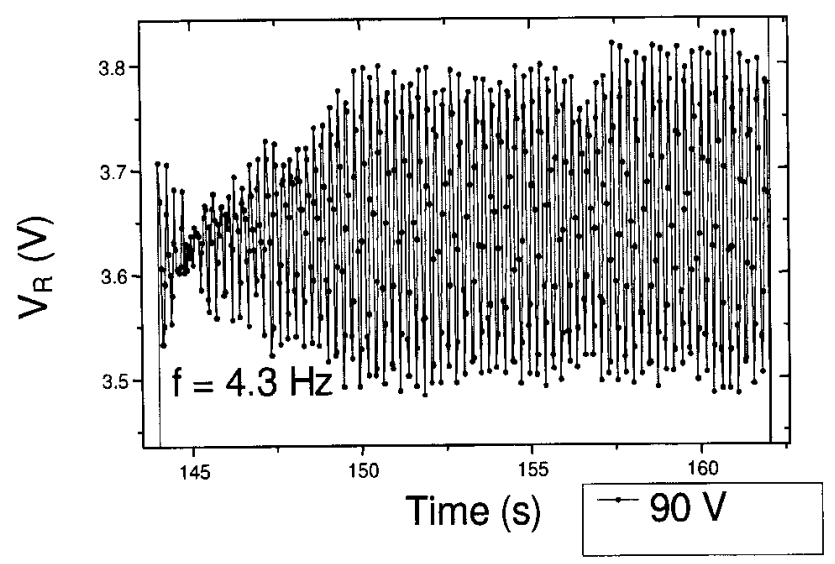

Figure 3. A zoom of Fig. 1 for the step of $90 \mathrm{~V}$. Observe that the main frequency is of $4.3 \mathrm{~Hz}$.

For measurements carried out at $2000 \mathrm{Vcm}^{-1}$ $(120 \mathrm{~V})$ we calculated the Fourier transform and the presence of many peaks were obtained. The main peak locates at frequency zeroTi.e. a constant term and the other peaks are listed in the table.

$\begin{array}{ll}\text { Frequency }(H z) & \text { RelativeIntensity } \\ 0 & \approx 1 \\ 2.8 & 0.0044 \\ 4.7 & 0.0049 \\ 5.6 & 0.066 \\ 10.8 & 0.011 \\ 11.3 & 0.017\end{array}$

If we neglect the two peaks at 10.8 and $11.3 \mathrm{~Hz}$ That at $2.8 \mathrm{~Hz}$ is the half of the frequency of the $5.6 \mathrm{~Hz}$ peaks. This fact is compatible with a bifurcation of cycle two.

In Fig. 4 we show the $\mathbf{j x E}$ characteristic as a function of the illumination intensity at $130 \mathrm{~K}$. The saturation of the sublinear behavior of the current density after the ohmic region is associated with field enhanced 
trapping in a deep level trap. Besides $\Gamma$ NC is observable for measurements using $30 \mathrm{~mA}$ trough the LED. Amplitude oscillations are easily observed in the sublinear regime and they increase with the electric field.

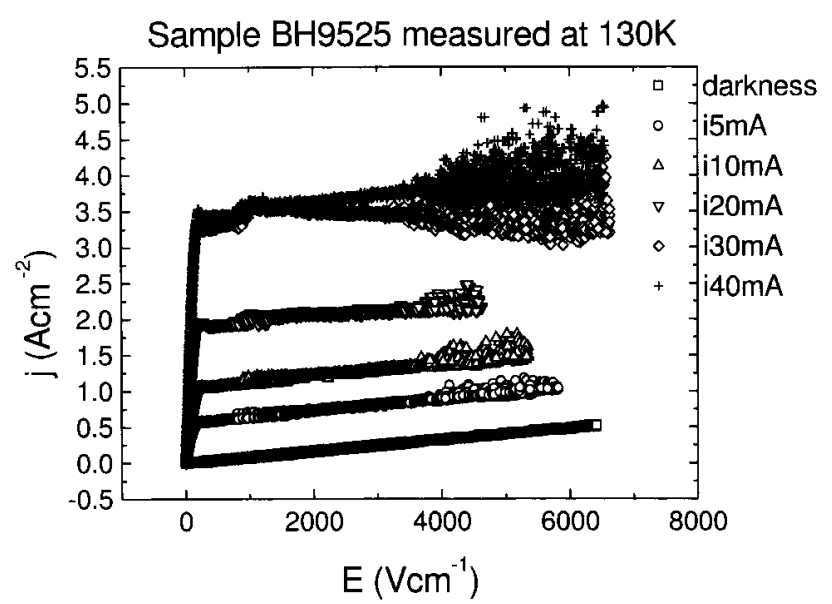

Figure 4. $j \times \mathbf{E}$ characteristic at $130 \mathrm{~K}$ as a function of the illumination intensity. The three like form of the curves is a kind of Poincare map of the LFO route to chaos.

In Fig. 5 we can observe a situation where the effect of field enhanced trapping is still stronger than those presented in Fig. 4. In this case $\Gamma$ the NDC region presents LFO but with amplitudes much smaller than those observed at $130 \mathrm{~K}$. Fig. 6 shows at $20 \mathrm{~K}$ an increasing efficiency in the field enhanced trapping and very complex LFO windows. Here the maximum of the current density for the highest illumination intensity is 5 times higher than the values observed at $100 \mathrm{~K}$.

\section{Sample BH9525 measured at 100K}

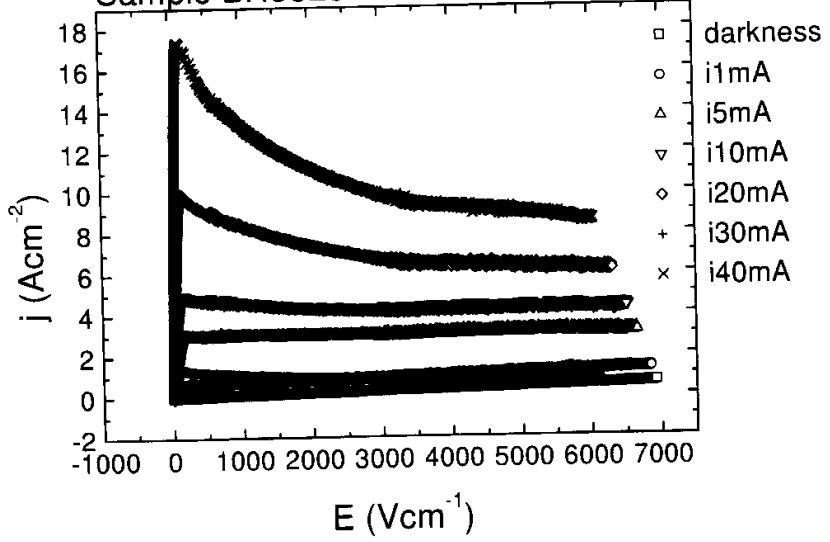

Figure 5. $j \times \mathbf{E}$ characteristic at $100 \mathrm{~K}$ as a function of the illumination intensity. At this temperature the field enhanced trapping effect is more effective, showing a $\mathrm{N}$ type NDC region for the three highest illumination intensity.

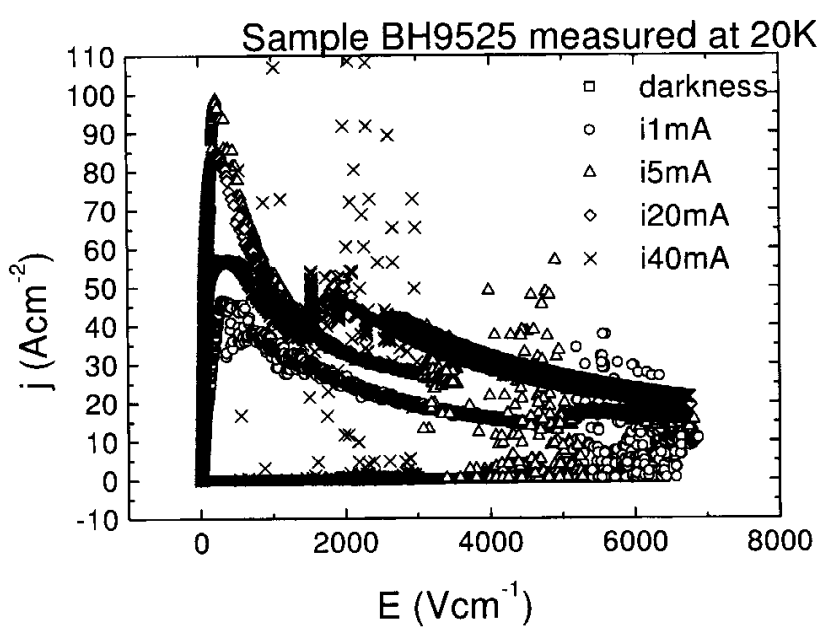

Figure 6. $j \times \mathbf{E}$ characteristic at $20 \mathrm{~K}$ as a function of the illumination intensity. At this temperatre the highest current is 5 times the highest current at $100 \mathrm{~K}$ and field enhanced trapping with LFO windows are presented.

\section{Discussion}

For the results shown in Fig. 1 we deduce that $\Gamma$ at room temperature and in darkness T the oscillation frequency is a function of the applied field. The amplitude also seems to increase with the field but only after a threshold of impurity breakdown. After a critical electric field $\Gamma$ which is between 1500 and $2000 \mathrm{Vcm}^{-1} \Gamma$ the LFO starts to present bifurcations of the main frequency. At room temperature we cannot obtain similar jxE characteristics to those found in the literature for the SI GaAs grown by liquid encapsulated Czochralski (LEC) [7]. The jxE characteristics are determined mainly by the impurity breakdown effect at this temperature. We know that the optical cross section and carrier life-time of deep trap present in our sample favor an increasing electron density for decreasing temperature and increasing light intensity. At $130 \mathrm{~K}$ however we reach the conditions that we have succeeded in obtaining similar data published in Ref. 7. Since our sample has a deep trap density of about three orders of magnitude higher than LEC samples we believe that the free carrier density rather than the impurity density is the important parameter in order to define improvements in the LFO characteristics.

For temperatures lower than $130 \mathrm{~K}$ field-enhanced trapping increases its dependence on the electrical field and a $\mathrm{N}$ shaped NDC region is observed. At this situation the intensity of the LFO are less strong when compared to the zero frequency term. Below $20 \mathrm{~K}$ we can see $\mathrm{LFO}$ windows in the $\mathbf{j x} \mathbf{E}$ characteristics. We believe that these effects are explained based on the fact that field-enhanced trapping is necessary in order to control 
the free carrier density in the $\Gamma$ valley of the conduction band. For a free carrier density high enough to form dipole domains of the Gunn type the field enhanced trapping could induce the recombination of the free electrons of the $\Gamma$ valley with ionized deep donors resulting in monopole domains which moves slower since the effective mass at the upper valleys of the conduction band is higher. Electrons in the $\mathrm{L}$ or $\mathrm{X}$ valleys recombine less efficiently with the deep level defects due to its differences in the $\mathrm{K}$ space. A low carrier density or a highly efficient field enhanced trapping effect (high temperature and below $130 \mathrm{~K}$ respectively) results in the destruction of the LFO monopoles before they reach the anode.

In summary we have measured LFO in an LTMBE GaAs sample grown at $300^{\circ} \mathrm{C}$ as a function of the electrical field $\Gamma$ temperature and illumination intensity. With these three parameters we were able to control the LFO characteristics. The electrical field controls the oscillation frequency the temperature and illumination control the carrier density and the field-enhanced trapping characteristic. We were able to identify how the LFO of our sample depends on the carrier density and on the power of field-enhanced trapping.

\section{References}

[1] G.N. Maracas and D.A. Johnson, Appl. Phys. Lett. 46, 305 (1985).

[2] E. Schöll, Phys. Rev. B 34, 1395 (1986).

[3] E. Schöll, Phys. Scrip. T29, 152 (1989).

[4] C. Backhouse, L. Young: Solid State Electr. 35, 1601 (1992).

[5] H.K. Sacks, A.G. Milnes: Int. J. Elect. 28, 565 (1970).

[6] S.P. McAlister, Z.-M. Li, D.J. Day: Canadian J. Phys. 69, 207 (1991).

[7] V.A. Samulov, proceedings of the Nonlinear Dynamics and Pattern Formation in Semiconductors and Devices, Springer, 220 (1995). 